\title{
Submitted: $\quad$ Multifocal fatty liver disease, insulin resistance \\ Accepted: \\ 13.10.2018 and carotid atherosclerosis: exploring the interrelated relationship
}

Published:

31.12.2018

\author{
Amr Shaaban Hanafy ${ }^{1}$, Sherief Abd-Elsalam², Ayman F. Ahmed³, \\ Mohammed M. Dawoud ${ }^{4}$
}

${ }^{1}$ Internal Medicine Department, Hepatogastroenterology Division, Zagazig University, Zagazig, Egypt ${ }^{2}$ Tropical Medicine Department, Division of Infectious Diseases, Tanta University, Tanta, Egypt

${ }^{3}$ Diagnostic Radiology Department, Zagazig University, Zagazig, Egypt

${ }^{4}$ Diagnostic Radiology Department, Tanta University, Tanta, Egypt

Correspondence: Dr. Amr Shaaban Hanafy, Internal Medicine Department, Hepatogastroenterology Division, Zagazig University, Sharkia, Zagazig, 44519, 40-Mostafa Fouad St., Egypt; tel.: +20110006186,e-mail: amrhanafy@zu.edu.eg

DOI: $10.15557 / \mathrm{JoU} .2018 .0044$

\section{Keywords}

focal fat,

insulin resistance, dyslipidemia, carotid atherosclerosis, systemic inflammation

\begin{abstract}
Introduction: Multifocal fatty liver infiltrations are not uncommon ultrasonographic finding; they are explained by the presence of aberrant vascular supply independent of the portal circulation or insulin resistance. Aim: To study the significance of this ultrasonographic finding. Methods: A study group ( $n=96)$ with multifocal fatty liver and two control groups: healthy subjects $(n=100)$ and patients with diffuse fatty liver disease $(n=100)$ were enrolled. They were tested for fasting blood glucose, lipid profile, transaminases, serum insulin, glycated hemoglobin, Homeostatic Model Assessment of Insulin Resistance, high-sensitivity C-reactive protein and liver stiffness in Fibroscan. Results: Patients with multifocal fatty liver showed a statistically significant higher values of serum transaminases, markers of insulin resistance, high-sensitivity C-reactive protein, and neutrophil lymphocyte ratio $(p<0.05)$. Lipid profile parameters were significantly higher $(p<0.05)$. Mean liver stiffness $(9.8 \pm 1.2 \mathrm{kPa})$ and carotid intima media thickness $(1.16 \pm 0.2 \mathrm{~mm})$ were significantly higher when compared to healthy subjects and patients with diffuse fatty liver disease. Independent predictors of insulin resistance and premature carotid atherosclerosis in patients with multifocal fatty liver disease were: serum gamma-glutamyl transferase (odds ratio 1.69), high-sensitivity C-reactive protein (odds ratio 1.62), uric acid (odds ratio 1.55), very low-density lipoprotein (odds ratio 1.74), total cholesterol/high-density lipoprotein (odds ratio 1.58) and severity of liver stiffness measured by Fibroscan (odds ratio 1.9). Conclusions: Multifocal fatty liver is an aggressive form of nonalcoholic fatty liver disease and should be considered a radiological sign of insulin resistance that needs special attention and management.
\end{abstract}

\section{Introduction}

In the next few years, fatty liver disease will become a new growing threat causing significant liver-related morbidity and mortality. It affects nearly $30 \%$ of the adult population and more than $50 \%$ of patients with diabetes and metabolic syndrome $\mathrm{e}^{(1,2)}$.

Hepatic steatosis represents the increased hepatic triglyceride (TG) content and may affect the whole liver or be present in a focal, circumscribed manner with areas of fat sparing ${ }^{(3)}$.It can be identified nearby specific locations, such as the gallbladder fossa, the subcapsular region, and the porta hepatis. Focal hepatic steatosis may be single or multiple, and attain a geographic appearance with the absence of a mass effect, so it represents a diagnostic challenge as it may be mistaken for a space-occupying lesion (a pseudotumor) and can be well-differentiated by magnetic resonance imaging $(\mathrm{MRI})^{(4,5)}$. 

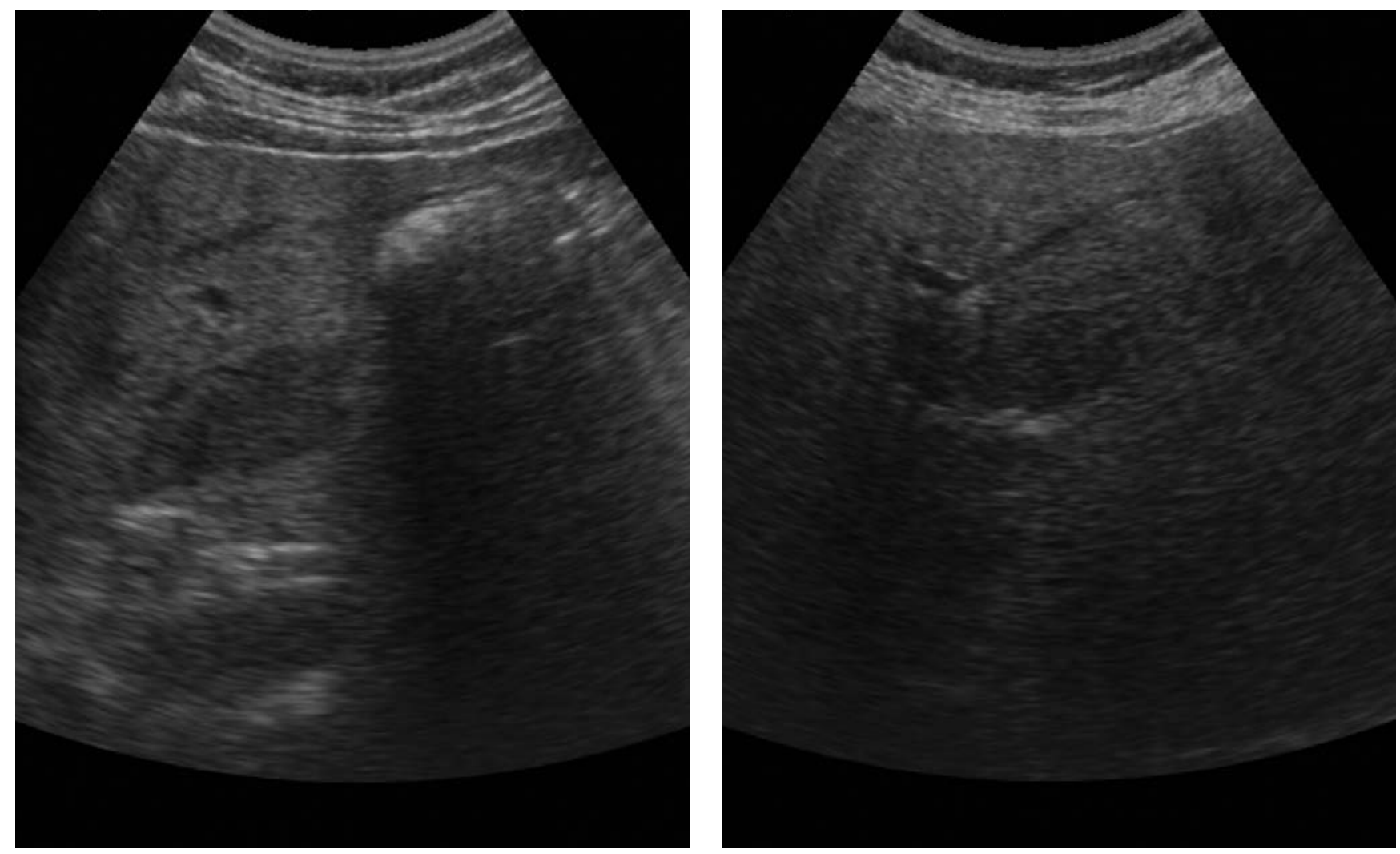

Fig. 1. Ultrasonography shows focal fatty sparing as a hypoechoic lesion in a steatotic liver

Abdominal computerized tomography scanning of a cohort of healthy adults revealed that fatty infiltration occurred in $9.7 \%$ of cases, $9 \%$ of which being focal and solitary, and $22 \%$ being focal and multiple. The prevalence increases with age and central obesity(6).

Solitary focal fatty liver is explained by the presence of aberrant vascular supply to the liver, independent of the portal circulation, that communicates with intrahepatic portal branches in a variable degree, such as the cholecystic veins, the epigastric-periumbilical veins ${ }^{(7)}$ and the parabiliary venous system of Couinaud, which drains the head of the pancreas ${ }^{(8)}$. This way the delivered concentrations of insulin are higher than the amount delivered by the portal venous system, leading to focal hepatic steatosis. Multifocal fatty liver could be explained by endothelial dysfunction associated with metabolic dysfunction ${ }^{(9)}$.

The atherogenic potential of fatty liver disease can be predicted if it is associated with hypertriglyceridemia, reduced high-density lipoprotein (HDL-C) and increased low-density lipoprotein (LDL) or abnormally increased VLDL ${ }^{(10,11)}$.

In insulin resistance (IR), the adipose tissue becomes resistant to anti-lipolytic effects of insulin with enhanced release of fatty acids leading to increased hepatic TG synthesis and lipotoxicity ${ }^{(12)}$.

The aim of the current work is to detect the clinical significance underlying the presence of the ultrasonographic find- ing of multifocal fatty liver disease and to find out if there is a difference from diffuse fatty liver disease regarding liver stiffness, lipid profile and enhanced systemic inflammation.

\section{Materials and methods}

\section{Patients selection}

An observational study was conducted in the period from May 2012 till August 2017. Ninety-six patients (10.1\%) who showed multifocal fatty liver disease (FFL) in abdominal ultrasonography (US) were selected from 952 patients with fatty liver disease and clinical high risk of cardio-metabolic syndrome, which included abdominal obesity with waist circumference over $88 \mathrm{~cm}$ in females and $102 \mathrm{~cm}$ in males, blood pressure $\geq 130 / 85 \mathrm{mmHg}$, or on anti-hypertensive medications, and body mass index $(\mathrm{BMI})>25^{(13)}$.

\section{Control groups}

A diffuse fatty liver group (DFL) $(n=100)$ and a group of healthy subjects $(n=100)$, matched for age and sex, were selected for comparison.

The patients were evaluated in the Hepatology Outpatient Clinic, Zagazig University and Tanta University hospitals in Egypt for right upper abdominal pain, gastroesophageal reflux, dyspepsia, or impaired blood glucose. 
Exclusion criteria included infection with HCV or HBV, drugs which induce steatosis (methotrexate, griseofulvin, tamoxifen, steroids, valproic acid and amiodarone) or insulin resistance (B blockers, steroids, immunosuppressive drugs, thiazide diuretics, antipsychotics as clozapine and risperidone), alcohol, pregnancy and smoking.

The study was approved by the ethical review board of the Faculty of Medicine of Zagazig University in April 2012 (IRB approval number: 3778/2012). Written consent was obtained from each patient included in the study before enrollment. The study protocol conforms to the ethical guidelines of the 1975 Declaration of Helsinki and its later amendments.

\section{Laboratory analysis}

Complete blood count was performed to detect neutrophil lymphocyte ratio (NLR), liver enzymes, such as ALT, AST, and GGT; the cut-off values selected were 30, 26, $29 \mathrm{U} / \mathrm{L}$ as they are associated with higher risk of steatosis-induced hepatocyte injury ${ }^{(14)}$.
High-sensitivity C-reactive protein (hsCRP) was measured by the high-sensitivity nephelometric method. The relative risk of cardiovascular events based on hsCRP was estimated as: low: CRP <1.0 mg/L; intermediate: CRP $1.0-3.0 \mathrm{mg} / \mathrm{L}$; and high: $\mathrm{CRP}>3.0 \mathrm{mg} / \mathrm{L}^{(15)}$.

Serum insulin was measured quantitatively by electrochemiluminescence immunoassay (ECLIA), the cut-off value was $8.64 \mu \mathrm{IU}$. Insulin resistance was calculated by Homeostatic Model Assessment of Insulin Resistance (HOMA-IR) using the following formula: fasting glucose (mg/dl) X fasting insulin $(\mu \mathrm{U} / \mathrm{ml}) / 405$.A value greater than 2 indicated insulin resistance ${ }^{(16)}$. Fasting blood glucose and $\mathrm{HbA}_{1 \mathrm{c}}$ were determined ${ }^{(17)}$.

Serum triglycerides (TGs), total cholesterol and HDL-C levels were determined using the spectrophotometric method after a 12-hour overnight fasting. LDL-C concentrations were calculated using the Friedewald formula: LDL-C $(\mathrm{mg} / \mathrm{dl})=\mathrm{TC}$ $(\mathrm{mg} / \mathrm{dl})$ - HDL-C (mg/dl) - TG (mg/dl)/5(18). VLDL cholesterol was based on the TGs by dividing the TGs by $5^{(19)}$. Total cholesterol /HDL ratio and LDL/HDL ratio were calculated ${ }^{(20)}$.
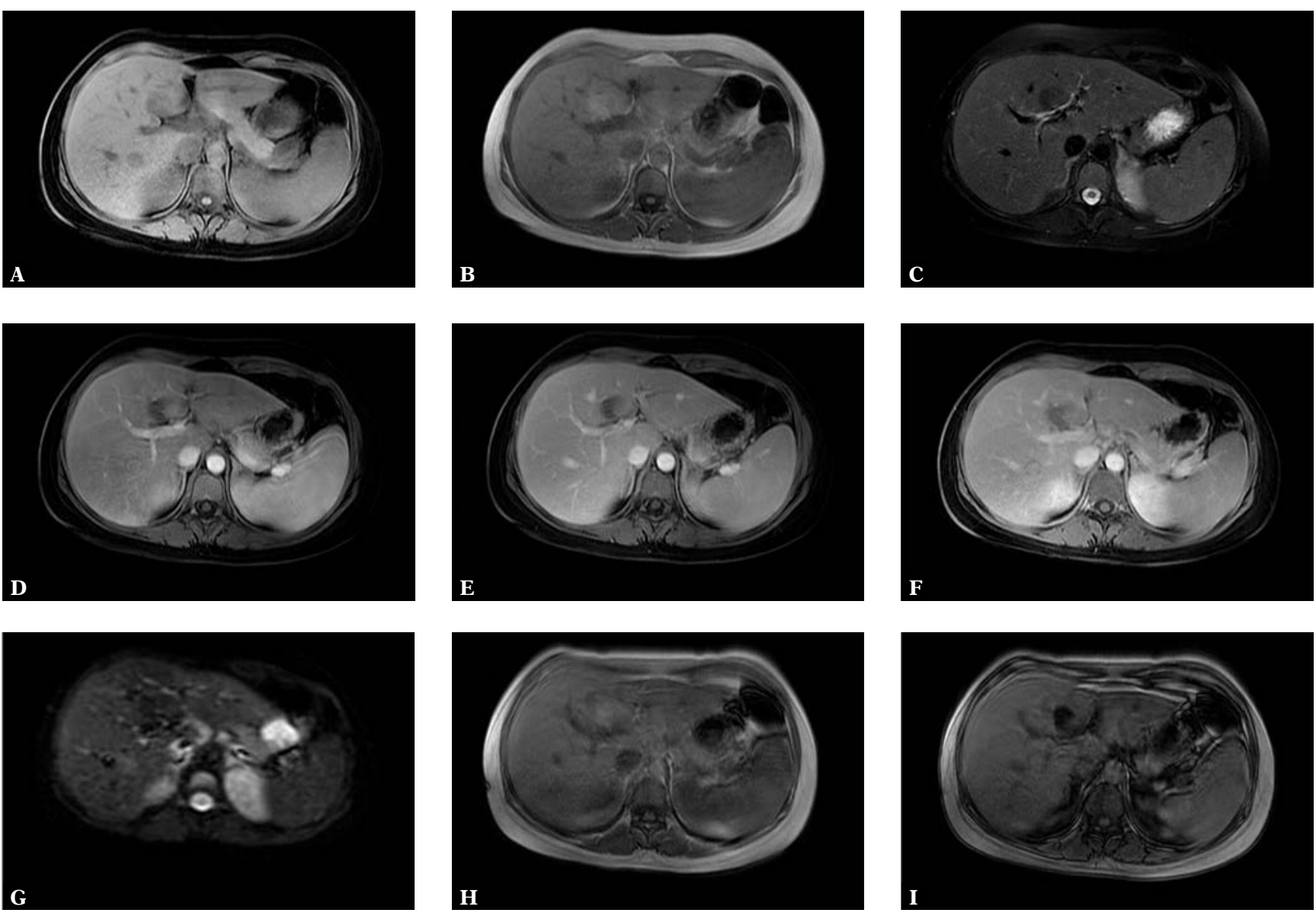

Fig. 2. Multiple focal hepatic lesions, the largest in segment IV measures about $34 \times 28 \mathrm{~mm}$. No enhancement in all phases of the examination, high signal intensity onT1WI, low signal intensity in the fat suppression sequence, and high signal intensity during in-phase study with a drop of signal intensity in out-of-phase study. A. Pre-contrast; B. T1W1; C. Fat suppression; D. Arterial phase; E. Venous phase; F. Delayed phase; G. DWI-diffusion weighted images; H. In-phase study; I. Out-of-phase study 
Biomarkers that correlated with hepatic steatosis included serum fasting uric acid and serum ferritin, which were determined quantitatively by ECLIA using Cobas e411immunoassay ${ }^{(21,22)}$.

\section{Abdominal ultrasonography (US)}

Abdominal ultrasound can accurately identify steatosis with a sensitivity of $94 \%$ and a specificity of $84 \%$ when steatosis affects more than $20 \%$ of the liver ${ }^{(23)}$. The scan was performed by one radiologist who was blinded for clinical data using Toshiba $77025 \mathrm{~A}$ with a convex probe of 3.5 MHz. Hepatic steatosis was defined as a diffuse increase of fine echoes in the liver parenchyma compared with that in the kidney based on standard criteria ${ }^{(24)}$. Focal fatty liver (FFL) disease was evaluated regarding the site, size and number of focal fatty lesions. They were differentiated from multiple hepatic hyperechoic metastases or multiple hemangiomas by triphasic dynamic magnetic resonance imaging using 1.5 Tesla.

\section{Liver stiffness measurement (LSM)}

Liver stiffness was measured by one physician who was blinded for clinical data, using FibroScan ${ }^{\circledR}$ (Echosens, Paris, France). The number of shots was 10 , success rate $\geq 60 \%$, interquartile range $\leq 25 \%$. It measures liver stiffness in a volume nearly 100 times bigger than liver biopsy. Liver stiffness of 2.5-7 kPa denotes F0-1, 7-9.5 kPa indicates F2, 9.5-12.5 $\mathrm{kPa}: \mathrm{F} 3$, and $>12.5 \mathrm{kPa}$ : cirrhosis. An XL probe was used ${ }^{(25)}$.

\section{Carotid artery intima-media thickness (CIMT)}

The common carotid arteries were scanned bilaterally via B-mode duplex ultrasound using a high-frequency $7.5 \mathrm{MHz}$ linear probe of Siemens G60® ultrasound system. The examination was performed by the same experienced radiologist who was blinded to the clinical and laboratory data of the participants. CIMT was measured as a distance from the intima-lumen interface to media-adventitia interface, the value over $0.9 \mathrm{~mm}$ was considered abnormal ${ }^{(26)}$.

\section{Liver biopsy}

Patients who presented with over doubled transaminase concentrations, liver stiffness $\geq 10 \mathrm{kPa}$, increased CIMT and agreed to the procedure, underwent US-guided liver biopsy of the largest lesion with hematoxylin-eosin staining for histological analysis and Masson trichrome staining for detection of fibrosis.

\section{Statistical analysis}

Data were analyzed using SPSS Statistics version 20 for Windows (SPSS Inc., Chicago, USA).Continuous variables were summarized as mean \pm standard deviation and standard of error (SE) when appropriate. Chi square test was used for categorical variables as frequency and percentage. Analysis of variance was appropriately used with post hoc analysis. Pearson' correlation coefficient and Spearman' rank correlations were used to assess a relationship between normally distributed and non-normally distributed risk variables, respectively. Logistic regression analysis was used to elucidate the independent relationships between IR, CIMT and focal fatty liver. Odds ratio was calculated from Exponential beta.

\section{Results}

Ninety-six patients with multifocal fatty liver infiltrations (FFL)were enrolled (Fig. 1). Their baseline demographic, laboratory and radiological characteristics were compared with 2 control groups whichincluded100healthy subjects (83 males, 17 females) and 100 patients with diffuse fatty liver (DFL) (80 males, 20 females) (Tab. 1).

A diagnosis of multifocal fatty liver disease was done by abdominal US, which revealed $12.7 \pm 4.3$ well-defined hyperechoic lesions scattered through all the liver. To differentiate them from multiple hepatic hyperechoic metastases or multiple hemangiomas, triphasic dynamic magnetic resonance imaging was done using 1.5 Tesla. On non-enhanced in-phase T1-weighted images, they were not detectable because of isointensity to the hepatic parenchyma. However, on out-of-phase T1-weighted images, the lesions appeared as hypointense areas, a finding that is highly consistent with a fatty component of these lesions. In addition, during delayed hepatobiliary phase images, the lesions were still recognized as well-defined hypointense foci in contrast to the adjacent liver tissue that showed higher signal intensity due to normal uptake of gadolinium (Fig. 2).

A statistically significant difference was found for BMI, being higher in the DFL and FFL groups when compared to the healthy controls, and a non-significant difference was observed between the DFF and FFL groups ( $p=0.19)$. AST, ALT and GGT were significantly higher in the FFL group when compared to the DFL group and healthy controls $(p=0.036,0.003,0.03)$, with no significant difference between DFL and healthy controls ( $p=0.062,0.2,0.12)$.

Markers of systemic inflammation, such as hsCRP and NLR, were significantly higher in the FFL group $(p=0.001,0.04)$ when compared to the DFL group and healthy controls, with a non-significant difference between healthy controls and the DFL group as regards NLR ( $p=0.34)$ (Tab. 1).

A significantly higher value of LSM was seen in Fibroscan in the FFL and DFL groups when compared to healthy controls $(p=0.001)$, with the examination performed in 10 patients. The difference between the FFL and DFL groups was non-significant $(p=0.19)$. Carotid intima-media thickness (CIMT) was significantly higher in the FFL and DFL groups when compared to healthy controls ( $p=0.023)$; it was higher in the FFL than DFL groups, but the difference was non-significant ( $p=0.21)$ (Tab. 1). 
Table 2 demonstrates the mean values of metabolic variables among the studied groups; fasting blood glucose (FBS) and insulin were significantly higher in the FFL patients ( $p=0.034,0.001$ respectively), with a non-significant difference between DFL and healthy controls $(p=0.068,0.16)$. $\mathrm{HbA}_{1 \mathrm{c}}$ and HOMA-IR were significantly higher in the FFL group ( $p=0.03,0.04$ ), with a non-significant difference between DFL and healthy controls $(p=0.15,0.21)$. Serum ferritin and uric acid were significantly higher in the FFL group ( $p=0.005,0.04)$, with a non-significant difference between the FFL and DFL ( $p=0.53,0.67$, respectively).

Serum TGs, total cholesterol (TC) and LDL-C were significantly higher in the FFL and DFL groups when compared to healthy controls, with higher values in patients with FFL than in those with DFL $(p=0.02,0.042,0.003)$. VLDL-C was significantly higher in the FFL group than in DFL and healthy controls $(p=0.02)$, with no difference between healthy controls and the DFL group ( $p=0.13)$ TC/ HDL ratio and LDL/HDL ratio were significantly higher in the FFL group than in the DFL group and healthy controls $(p=0.001,0.02)$. They were also significantly higher in the DFL patients than in healthy controls $(p=0.002,0.001)$.

Patients who showed more than a double increase in serum transaminases and $\mathrm{LSM} \geq 10 \mathrm{kPa}$, and after their approval $(n=10)$, underwent percutaneous liver biopsy which demonstrated no evidence of neoplasia, mixed micro- and macrovesicular steatosis with lymphocyte cell infiltrates $(n=10)$, ballooning degeneration $(n=4)$, and fibrous septa $(n=6)$.

The cutoff value of LSM in Fibroscan, associated with histological severity of focal fatty liver was $8 \mathrm{kPa}$ with sensitivity $94 \%$, specificity 95\%, AUC 0.845 ; 95\% CI 0.93-0.97. The cutoff value of LSM in FFL associated with IR was9.3 $\mathrm{kPa}$ with sensitivity 93.1\%, specificity 97.9\%, AUC0.93; 95\% CI 0.96-0.99, as shown in Fig. 3.

The variables that correlated with the severity of LSM in Fibroscan, and so had fibrogenic potential, were: GGT $(r=0.454, p=0.001)$, AST $(r=0.335, p=0.002)$, ferritin $(r=0.553, p=0.000), \mathrm{TC} / \mathrm{HDL}(r=0.346, p=0.001)$, and VLDL-C $(r=0.256, p=0.004)$.

The occurrence of IR and premature carotid atherosclerosis in the FFL group were significantly correlated with: GGT $(r=0.654, p=0.000)$, hsCRP $(r=0.635, p=0.000)$, NLR $(r=0.439, p=0.001)$, uric acid $(r=0.548$, $p=0.000)$, ferritin $(r=0.453, p=0.000)$, triglycerides $(r=0.321, p=0.000), \mathrm{LDL}(r=0.534, p=0.000), \mathrm{TC} / \mathrm{HDL}$ $(r=0.546, p=0.001), \mathrm{LDL} / \mathrm{HDL}(r=0.501, p=0.001)$, VLDL-C $(r=0.456, p=0.000)$, and LSM in Fibroscan $(r=0.645, p=0.000)$.

Independent predictors of IR and premature carotid atherosclerosis in the FFL group were: serum GGT $(\beta=0.402$, $p=0.000$, odds ratio (OR) 1.69), hsCRP $(\beta=0.390$, $p=0.000$, OR 1.62), uric acid $(\beta=0.323, p=0.000, \mathrm{OR}$ $1.55)$, VLDL-C $(\beta=0.424, p=0.000$, OR 1.74), TC/HDL $(\beta=0.389, p=0.000$, OR 1.58), and severity of LSM in Fibroscan $(\beta=0.490, p=0.000$, OR 1.9).

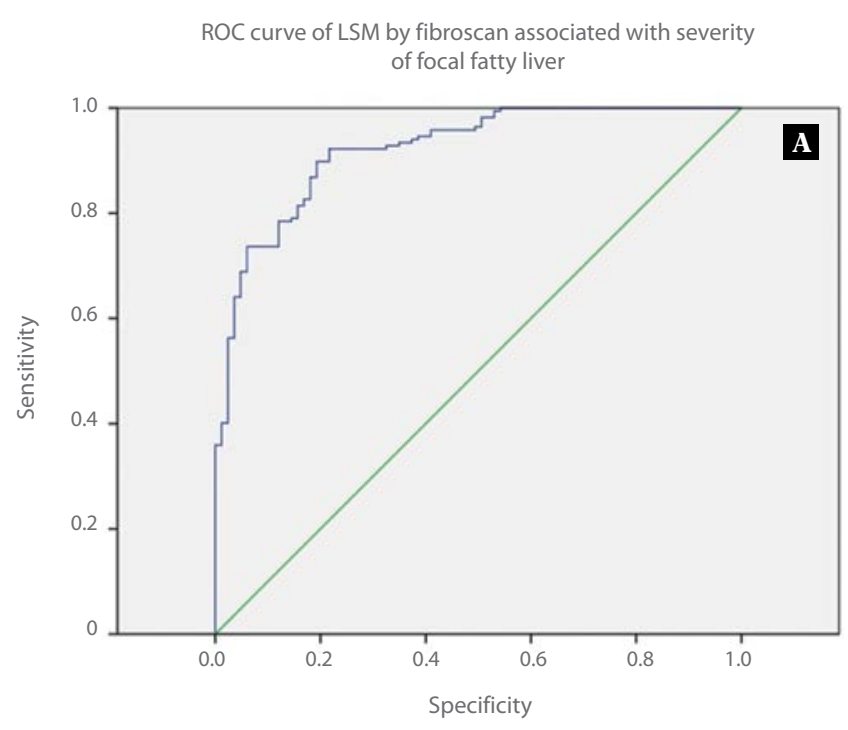

ROC curve of LSM by fibroscan associated with insulin resistance (IR)

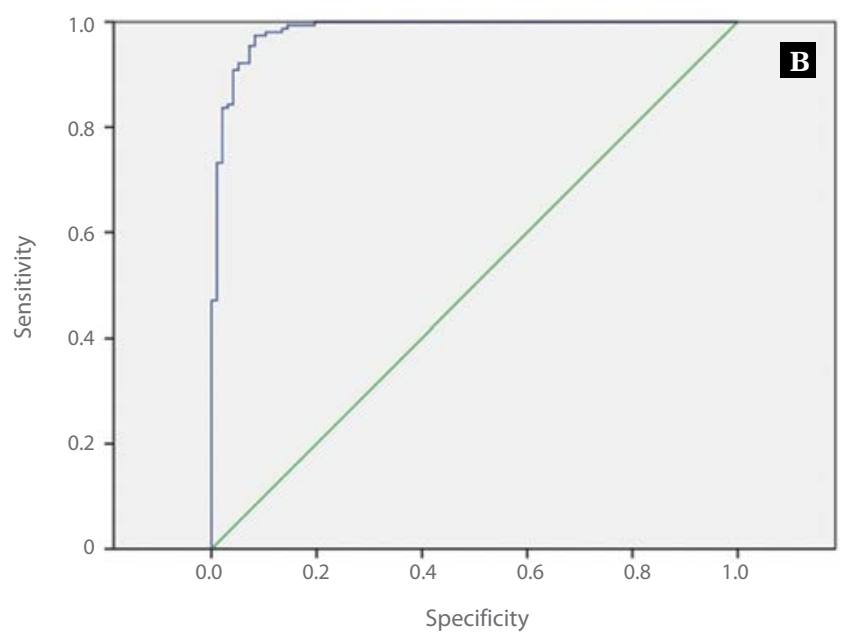

Fig. 3. ROC curve of the cutoff value of LSM associated with histological severity of focal fatty liver (A) and insulin resistance $\mathbf{( B )}$

\section{Discussion}

Focal hepatic steatosis could be mistaken for metastatic or infiltrative deposits. Nevertheless, itis recognized on the basis of the typical distribution of lesions and the noninvasion of the nearby blood vessels ${ }^{(27,28)}$.

Multifocal fatty infiltrations are associated with metabolic derangement, mainly IR or type 2 diabetes, with accentuated inflammatory response. This is proven by the fact that focality is initiated by endothelial dysfunction, and local ischemia is induced by hyperinsulinemia, which further accentuates hepatic steatosis due to impairment in a phosphatidylinositol 3-kinase-dependent pathway that will lead to an imbalance between nitric oxide and endothelin- $1^{(29)}$. In IR, gluconeogenesis and lipogenesis are enhanced due 


\begin{tabular}{|c|c|c|c|c|}
\hline Variable & $\begin{array}{c}\text { Diffuse } \\
\text { fatty liver }\end{array}$ & $\begin{array}{c}\text { Focal } \\
\text { fatty liver }\end{array}$ & $\begin{array}{l}\text { Healthy } \\
\text { controls }\end{array}$ & $p$ \\
\hline$N$ & 100 & 96 & 100 & $0.6^{*}$ \\
\hline $\operatorname{Sex}(M / F)$ & $80 / 20$ & $70 / 26$ & $83 / 17$ & $0.23^{*}$ \\
\hline Age & $36.7 \pm 1.8$ & $37.6 \pm 2.7$ & $36 \pm 0.2$ & 0.17 \\
\hline BMI $\left(\mathbf{k g} / \mathbf{m}^{2}\right)$ & $28.3 \pm 1.4$ & $28.9 \pm 2.6$ & $24.3 \pm 1.1$ & 0.03 \\
\hline AST (IU/I) & $32.7 \pm 4.6$ & $77.5 \pm 28.4$ & $26 \pm 3$ & 0.036 \\
\hline ALT (IU/I) & $37.3 \pm 4$ & $88.2 \pm 33.7$ & $27 \pm 5$ & 0.003 \\
\hline GGT (IU/I) & $30.2 \pm 4.6$ & $57.8 \pm 20$ & $23 \pm 5$ & 0.03 \\
\hline Albumin (g/dl) & $4.3 \pm 0.5$ & $4.4 \pm 0.2$ & $4.5 \pm 0.4$ & 0.1 \\
\hline Total bilirubin (mg/dl) & $1 \pm 0.1$ & $1.12 \pm 0.1$ & $0.9 \pm 0.1$ & 0.2 \\
\hline HsCRP (mg/l) & $2.3 \pm 0.85$ & $4.8 \pm 1.1$ & $0.66 \pm 0.35$ & 0.001 \\
\hline NLR & $1.6 \pm 0.1$ & $2.7 \pm 0.7$ & $1.56 \pm 0.4$ & 0.04 \\
\hline FibroScan (kPa) & $9.6 \pm 0.9$ & $9.8 \pm 1.2$ & $\begin{array}{c}3.6 \pm 1.2 \\
\text { (10 patients) }\end{array}$ & 0.01 \\
\hline CIMT (mm) & $1.02 \pm 0.1$ & $1.16 \pm 0.2$ & $0.88 \pm 0.05$ & 0.023 \\
\hline
\end{tabular}

Tab. 1. Baseline laboratory, metabolic and Fibroscan values of patient subgroups

\begin{tabular}{|c|c|c|c|c|}
\hline Variable & $\begin{array}{c}\text { Diffuse } \\
\text { fatty liver } \\
\text { ( } n=100\end{array}$ & $\begin{array}{c}\text { Focal } \\
\text { fatty liver } \\
(n=96)\end{array}$ & $\begin{array}{l}\text { Healthy } \\
\text { controls } \\
(n=100)\end{array}$ & $p$ \\
\hline FBS (mg/dl) & $93.5 \pm 5.3$ & $105 \pm 20$ & $82 \pm 6$ & 0.034 \\
\hline Insulin & $6.1 \pm 0.7$ & $9.3 \pm 1.8$ & $5.1 \pm 0.3$ & 0.001 \\
\hline $\mathrm{HbA}_{1 \mathrm{c}} \%$ & $5.2 \pm 0.37$ & $6.7 \pm 0.9$ & $4.4 \pm 0.37$ & 0.03 \\
\hline HOMA-IR & $1.4 \pm 0.2$ & $2.64 \pm 0.6$ & $1.3 \pm 0.3$ & 0.04 \\
\hline Ferritin (ng/dl) & $326 \pm 18.7$ & $352.3 \pm 81.1$ & $183 \pm 13$ & 0.005 \\
\hline Uric acid (mg/dl) & $6.5 \pm 0.4$ & $6.8 \pm 1.6$ & $4.2 \pm 0.6$ & 0.04 \\
\hline TGs mg/dl & $143.7 \pm 21.3$ & $173 \pm 10.8$ & $122 \pm 9$ & 0.02 \\
\hline LDL mg/dl & $157 \pm 12.1$ & $220.3 \pm 13.3$ & $99.2 \pm 8.9$ & 0.003 \\
\hline HDL (mg/dl) & $37.3 \pm 4.9$ & $35.7 \pm 3.2$ & $44.3 \pm 6.2$ & 0.3 \\
\hline VLDL (mg/dl) & $27.8 \pm 6.9$ & $34.7 \pm 4.3$ & $23.7 \pm 5.6$ & 0.02 \\
\hline Total cholesterol & $220.4 \pm 18.5$ & $292.6 \pm 12.4$ & $173.6 \pm 11.5$ & 0.032 \\
\hline TC/HDL ratio & $5.72 \pm 1.9$ & $7.8 \pm 1.4$ & $3.7 \pm 0.9$ & 0.001 \\
\hline LDL/HDL ratio & $4.33 \pm 2.6$ & $5.9 \pm 2.4$ & $2.39 \pm 0.7$ & 0.02 \\
\hline
\end{tabular}

Tab. 2. The mean values of the metabolic variables among the studied groups 
to direct activation of sterol regulatory element-binding protein 1 (SREBP-1) transcription factor ${ }^{(30)}$.

Endothelial dysfunction is an important variable for the occurrence of atherosclerosis. CIMT represents an important radiological sign of subclinical atherosclerosis and may predict the future occurrence of ischemic heart disease and cerebrovascular stroke ${ }^{(31)}$. The correlation between focal fatty infiltrations and carotid atherosclerosis had not been studied before. In general, nonalcoholic fatty liver disease (NAFLD) is associated with persistent inflammation with increased risk of subclinical carotid atherosclerosis ${ }^{(32)}$, and histological progression in NAFLD is independently associated with increasing CIMT $^{(33)}$.

The current study was conducted in a group of 96 patients who presented with multifocal fatty liver disease as a subtype of NAFLD diagnosed by US and confirmed by dynamic triphasic MRI.

The significance of this radiological entity was investigated in comparison to diffuse fatty liver disease and healthy controls,. FFL could be the early aggressive form of NAFLD. This is represented by a statistically significant higher values of AST, ALT, GGT and Fibroscan value $(9.8 \pm 1.2 \mathrm{kPa})$, corresponding to F2-3, reflecting the fibrogenic nature of these lesions. This was confirmed by liver biopsy, despite the fact that some patients with diagnosed nonalcoholic steatohepatitis (NASH) may have normal serum transaminases ${ }^{(34)}$.

Markers of insulin resistance, such as serum insulin and HOMA-IR, and markers of subclinical inflammation, such as hsCRP and NLR, were significantly higher in the FFL patients than in other subgroups. Hs-CRP can be used as a non-invasive marker of inflammatory response in NAFLD and a predictor of future occurrence of NAFLD in healthy adults with high normal values ${ }^{(35,36)}$. NLR is an effective and inexpensive marker that is better than $\mathrm{C}$-reactive protein when correlated with histological progression in $\mathrm{NASH}^{(37)}$.

Metabolic biomarkers, such as serum ferritin and uric acid, were significantly higher in the FFL group, and this

\section{References}

1. Sayiner M, Koenig A, Henry L, Younossi ZM: Epidemiology of nonalcoholic fatty liver disease and nonalcoholic steatohepatitis in the United States and the rest of the world. Clin Liver Dis 2016; 20: 205-214.

2. Calzadilla Bertot L, Adams LA: The natural course of non-alcoholic fatty liver disease. Int J Mol Sci 2016; 17: pii: E774.

3. Kawano Y, Cohen DE: Mechanisms of hepatic triglyceride accumulation in non-alcoholic fatty liver disease. J Gastroenterol 2013; 48: 434-441.

4. Hamer OW, Aguirre DA, Casola G, Lavine JE, Woenckhaus M, Sirlin CB: Fatty liver: imaging patterns and pitfalls. Radiographics 2006; 26: 1637-1653.

5. Tom WW, Yeh BM, Cheng JC, Qayyum A, Joe B, Coakley FV: Hepatic pseudotumor due to nodular fatty sparing: The diagnostic role of opposed-phase MRI. Am J Roentgenol 2004; 183: 721-724.

6. el-Hassan AY, Ibrahim EM, al-Mulhim FA, Nabhan AA, Chammas MY: Fatty infiltration of the liver: analysis of prevalence, radiological and clinical features and influence on patient management. Br J Radiol 1992; 65: 774-778. proves the fact that serum uric acid and ferritin have an interrelated pathogenic pathway and are associated with endothelial dysfunction, which favors the development of hepatic steatosis in metabolic syndrome ${ }^{(38)}$.

TGs, VLDL, TC/HDL and LDL/HDL were significantly higher in the FFL group. Higher LDL is associated with an increased risk of NAFLD, even in non-obese patients ${ }^{(39)}$, and patients who show high TC/HDL or TG/HDL-ratios have a higher risk for NAFLD ${ }^{(40-42)}$.

To our knowledge, no study has searched for the occurrence of atherogenic dyslipidemia in multifocal fatty infiltration or its potential hazards, or underlying metabolic risks.

In conclusion, this research provided significant information about a neglected subtype of NAFLD, characterized by multifocal hepatic fatty deposition which may indicate or herald the occurrence of more advanced histological features and should be considered an aggressive form of NASH. It is also associated with a higher risk of premature atherosclerosis. FFL should be considered a radiological sign of insulin resistance, which needs specific attention and care.

\section{Acknowledgement}

I would like to thank the staff of the Clinical Pathology and Diagnostic Radiology Departments in Zagazig and Tanta Universities for helping in the current study.

\section{Conflict of interest}

Authors do not report any financial or personal connections with other persons or organizations, which might negatively affect the contents of this publication and/or claim authorship rights to this publication.

Dr Amr Hanafy is the guarantor of this work and, as such, had full access to all the data in the study and takes responsibility for the integrity of the data and the accuracy of the data analysis.
7. Yoshimitsu K, Honda H, Kuroiwa T, Irie H, Aibe H, Shinozaki K et al.: Unusual hemodynamics and pseudolesions of the noncirrhotic liver at CT. Radiographics 2001; 21: S81-S96.

8. Couinaud C: The parabiliary venous system. Surg Radiol Anat 1988; 10: 311-316.

9. Khalili K, Lan FP, Hanbidge AE, Muradali D, Oreopoulos DG, Wanless IR: Hepatic subcapsular steatosis in response to intraperitoneal insulin delivery: CT findings and prevalence. Am J Roentgenol 2003; 180: 1601-1604.

10. Sonmez A, Nikolic D, Dogru T, Ercin CN, Genc H, Cesur M et al.: Lowand high-density lipoprotein subclasses in subjects with nonalcoholic fatty liver disease. J Clin Lipidol 2015; 9: 576-582.

11. Jiang ZG, Tapper EB, Connelly MA, Pimentel CF, Feldbrügge L, Kim M et al.: Steatohepatitis and liver fibrosis are predicted by the characteristics of very low density lipoprotein in nonalcoholic fatty liver disease. Liver Int 2016; 36: 1213-1220.

12. Arner P: Insulin resistance in type 2 diabetes: role of fatty acids. Diabetes Metab Res Rev 2002; 18 (Suppl. 2): S5-S9. 
13. Grundy SM, Cleeman JI, Daniels SR, Donato KA, Eckel RH, Franklin BA et al.: Diagnosis and management of the metabolic syndrome: An American Heart Association/National Heart, Lung, and Blood Institute Scientific Statement. Circulation 2005; 112: 2735-2752.

14. Prati D, Taioli E, Zanella A, Della Torre E, Butelli S, Del Vecchio E et al.: Updated de nitions of healthy ranges for serum alanine aminotransferase levels. Ann Intern Med 2002; 137: 1-10.

15. Pearson TA, Mensah GA, Alexander RW, Anderson JL, Cannon RO 3rd, Criqui M et al.: Markers of inflammation and cardiovascular disease. Ap- plication to clinical and public health practice: A statement for healthcare professionals from the Centers for Disease Control and Prevention and the American Heart Association. Circulation 2003; 107: 499-511.

16. Matthews DR, Hosker JP, Rudenski AS, Naylor BA, Treacher DF, Turner RC: Homeostasis model assessment: Insulin resistance and beta-cell function from fasting plasma glucose and insulin concentrations in man. Diabetologia 1985; 28: 412-419.

17. American Diabetes Association: Diagnosis and classification of diabetes mellitus. Diabetes Care 2010; 33: S62-S69. 